\title{
Endovascular delivery of leads and stentrodes and their applications to deep brain stimulation and neuromodulation: a review
}

\author{
Gary Rajah, MD, ${ }^{1,3}$ Hamidreza Saber, MD, ${ }^{2,3}$ Rasanjeet Singh, BA, ${ }^{3}$ and \\ Leonardo Rangel-Castilla, MD4
}

Departments of ${ }^{1}$ Neurosurgery and ${ }^{2}$ Neurology, Wayne State University, and ${ }^{3}$ Wayne State University School of Medicine, Detroit, Michigan; and ${ }^{4}$ Department of Neurosurgery, Mayo Clinic, Rochester, Minnesota

\begin{abstract}
Neuromodulation and deep brain stimulation (DBS) have been increasingly used in many neurological ailments, including essential tremor, Parkinson's disease, epilepsy, and more. Yet for many patients and practitioners the desire to utilize these therapies is met with caution, given the need for craniotomy, lead insertion through brain parenchyma, and, at many times, bilateral invasive procedures. Currently endovascular therapy is a standard of care for emergency thrombectomy, aneurysm treatment, and other vascular malformation/occlusive disease of the cerebrum. Endovascular techniques and delivery catheters have advanced greatly in both their ability to safely reach remote brain locations and deliver devices. In this review the authors discuss minimally invasive endovascular delivery of devices and neural stimulating and recording from cortical and DBS targets via the neurovascular network.
\end{abstract}

https://thejns.org/doi/abs/10.3171/2018.4.FOCUS18130

KEYWORDS deep brain stimulation; stentrode; endovascular surgery

$\mathrm{N}$ EUROSTIMULATION has proven effective for many diseases, including Parkinson's disease ${ }^{11}$ and essential tremor, ${ }^{23}$ and has shown promising results for newer targets, including chronic pain, obsessive-compulsive disorder, ${ }^{19}$ refractory depression, ${ }^{34}$ and, most recently, neurocognitive disorders..$^{12,37}$ As advances in neuromodulation continue, its promise grows in remedying various neurological ailments. At this time, deep brain stimulation (DBS) devices consist of surgically implanted leads passed, using the assistance of neuronavigation, through the cortical surface to rest in deep brain structures. MRI, awake-craniotomy procedures, or microelectrode recordings are utilized to ensure that the lead penetrates the correct area of the neural network. The lead is then tunneled under the galea and connected to a generator implanted subcutaneously. While DBS systems have greatly advanced in electrode design and patterns of stimulation, ${ }^{20}$ common DBS systems today such as the Activa PC (Medtronic) still rely on a transcranial lead connected to a battery. Wireless systems have been proposed in theory and reported on, but they still necessitate a transcranial lead. ${ }^{28}$ Nonincisional alternatives to DBS also exist: techniques such as focused ultrasound ${ }^{30}$ and Gamma Knife radiosurgery ${ }^{26}$ provide therapeutic results via a nonsurgical lesionectomy within their targets. One drawback of these noninvasive therapies is the finality of the lesion that is created. Even these nonsurgical therapies, however, can result in unwanted neurological deficits. One study of 209 patients in whom the Gamma Knife was used to treat the ventral intermediate thalamic nucleus reported a $6.9 \%$ rate of both transient and permanent complications. ${ }^{58}$

While the complications of DBS are low in comparison to other procedures, there is risk for permanent neurological damage. ${ }^{13}$ Long-term hardware complication rates associated with DBS are reported to be $8.4 \%$ per electrode/ year. ${ }^{39}$ Major surgical complication rates for DBS lead insertion include a $3.3 \%$ risk of hematoma of any type per lead implanted and a $0.6 \%$ risk of major neurological deficits. Hemorrhage rates have been shown to correlate with the number of electrode passes. ${ }^{2}$ Thus, while DBS systems

ABBREVIATIONS DBS = deep brain stimulation; EEG = electroencephalographic; PED = Pipeline embolization device.

SUBMITTED March 16, 2018. ACCEPTED April 17, 2018.

INCLUDE WHEN CITING DOI: 10.3171/2018.4.FOCUS18130. 
continue to advance in design, safety, and effective modulation of their chosen target, new techniques utilizing minimally invasive lead placement should be trialed. This review will discuss the progress made in endovascular lead delivery, stentrodes (stents with the ability to record or stimulate surrounding tissue), and their application to DBS, responsive neural stimulation, and neuromodulation.

\section{Endovascular Delivery of Leads}

Many intracranial pathologies are treated using catheter-based techniques utilizing the vascular network largely via a transfemoral artery or vein approach. Stents are delivered for the treatment of wide-necked aneurysms, ${ }^{3}$ stenosis,${ }^{17}$ and repair of vessels. Similarly coils, particles, and liquid embolic agents are also delivered into the intracerebral vessels for the treatment of aneurysms, arteriovenous malformations, arteriovenous fistulas, and hypervascular tumors. There are now numerous guide catheters, intermediate catheters, and microcatheters or wires capable of safely delivering devices of many sizes in awake ${ }^{44}$ or anesthetized patients. Benefits of endovascular delivery of devices include avoiding craniotomy, faster recovery times, and possibly less patient anxiety. There are numerous patents listed for endovascular cranial lead insertion, ${ }^{52}$ stentbased lead anchors, ${ }^{4}$ and disease-specific systems ${ }^{53}$ An interesting article by Robert Ritter published in 2017 (https:// www.linkedin.com/pulse/neurovascular-approach-deepbrain-stimulation-robert-ritter-iii) describes a submillimeter nitinol stent anchoring 100 microwires of $0.2-\mu$ thickness capable of accessing very thin capillaries. Watanabe et al. have further studied their vascular microelectrode array in an intravascular spinal cord recording in Xenopus laevis (frog) ${ }^{54}$ The electrode material described in the Ritter article proposes iridium oxide, which was initially described by Cogan et al.. ${ }^{10}$ to increase charge injection, given the small lead size. Wong et al. have also evaluated nitinol electrodes intravascularly for the measurement of visual evoked potentials in sheep. ${ }^{56}$

Endovascular placement of leads has successfully allowed for multicellular recording of brain activity. ${ }^{56}$ This type of recording or stimulation is necessary as brain activity is composed of coherent multicellular responses best understood via simultaneous recordings from numerous locations. As capillaries permeate every aspect of the brain, creating approximately $25,000 \mathrm{~m}$ of vessel, an endovascular approach toward neuromodulation with $10-\mu$ vessels coursing in the vicinity of individual neurons, provides a vast network of untapped potential..$^{32}$ Ritter hypothesized that the high impedance from endovascularly placed leads (given the decreased lead size) could be overcome by the close proximity of the lead to undamaged neural elements. Discussion of all the possible nanoscale polymers and metal able to create capillary or venulecompatible leads is outside the scope of this review.

This approach has limitations. While stimulation parameters would need to be identified, the placement of many electrodes could provide theoretical advantages in accuracy. Vessel injury or thrombosis is a concern as with any intravascular device, but this would be less of a concern given the small size of such devices. Our best educated guess on device-related effects can be gleaned from Pipeline embolization device (PED; Medtronic) data on flow diversion for the treatment of aneurysms. Metaanalysis data on the PED identified a 5\% procedure-related morbidity and mortality rate among 1451 patients. Perforator infarction occurred in 3\% of patients, and subarachnoid hemorrhage and intraparenchymal hemorrhage in 3\% each. The ischemic stroke rate was 6\% (lower in the anterior circulation and higher in the posterior circulation). ${ }^{7}$ One caveat to the aforementioned data is that the amount of metal coverage is $33 \%-35 \%$ for PEDs, which is very high, likely indicating the uppermost level of risk for stroke or occlusion. Since the stentrode would not be placed for an aneurysm, the risk of aneurysm rupture would be absent. Neuroform stents used for stent-assisted coiling of aneurysms have $6 \%$ metal coverage (much less than the PED). One study examining thromboembolic risks in these stents reported a $0.5 \%$ thromboembolism risk (13/237 patients), with 2 patients having late in-stent stenosis. ${ }^{18}$ LVIS (low profile visualized intraluminal support) and LVIS Jr. devices have 18\%-22\% metal coverage, and Iosif et al. reported a $2.2 \%$ event rate of sequelae related to the stent. ${ }^{24}$ Antiplatelets remain an option, such as aspirin or dual antiplatelet therapy, as currently prescribed for patients receiving intravascular stents. Trials involving stentrodes, as discussed in a later section, have shown similar coagulation profiles to modern stents, ${ }^{16}$ further reinforcing the possibility of similar management. New stent coatings are also on the horizon, credited with decreasing the amount of thrombogenicity. One such coating is the SHIELD technology, a phosphorylcholine coating for PEDs. ${ }^{41}$ Similar coating technology may prove useful for neurovascular lead design. The stents of today may look nothing like the stents of the future, especially as stentrodes will not have to support coils, hold open plaques, and so on. The structural properties could vary greatly compared to present-day devices.

Achieving an accurate therapeutic location for stentrode placement and the need for retreatment are also concerns. Stent placements can be done in awake patients with intraoperative physiological testing prior to stent deployment. Currently PEDs are deployed in awake patients, ${ }^{44}$ and super-selective awake WADA testing can be performed, or test electrodes may be utilized to determine if the desired physiological response is present prior to permanent stent placement. While not optimal, currently stents can be placed over one another when necessary, as in the case with recurrent stent-coiled aneurysms treated with PED flow diversion. ${ }^{9}$ Last, anatomical vessel variation may preclude some stentrode placements. Awake physiological testing during angiography will help identify these patients in whom a stentrode should not be placed.

\section{Feasibility of Neurovascular Array Efficacy for Target Disease}

Studies of intravascular neuromodulation for both recording and stimulation have described a variety of potential targets for therapeutic management (Table 1). ${ }^{25,27,37}$, 42,46,49 Teplitzky et al. ${ }^{49}$ identified 17 potential DBS targets utilizing a computational model that assessed targets ideal 
TABLE 1. Potential vascular targets for recording or stimulating neural targets

\begin{tabular}{|c|c|c|c|}
\hline Target Location & Vessel & Previously Described & Vessel or Device Size \\
\hline $\begin{array}{l}\text { Motor or sensory strip; other cortical } \\
\text { convexity area depending on } \\
\text { MRV or angiography findings }\end{array}$ & $\begin{array}{l}\text { Cortical vein or petrosquamosal branch } \\
\text { of the middle meningeal artery }\end{array}$ & $\begin{array}{l}\text { Oxley et al., 2016; Stoeter et al., } \\
\text { 1995: EEG recording }\end{array}$ & $1-5 \mathrm{~mm}^{*}$ \\
\hline Mesial temporal structures & Cavernous sinus \& petrosal sinus & Kunieda et al., 2000, EEG recording & $\begin{array}{l}\text { Utilized a Seeker Lite-10 } \\
\text { guidewire electrode }\end{array}$ \\
\hline Lateral temporal structures & Middle meningeal artery & Ishida et al., 1998, EEG recording & Microwire utilized \\
\hline Brainstem & Basilar artery & \multirow[t]{4}{*}{ Stoeter et al., 1995, EEG recording } & \multirow{4}{*}{$\begin{array}{l}\text { Microguidewire, poly-teflor } \\
\text { insulated }\end{array}$} \\
\hline Parietal lobe & Middle cerebral artery branch & & \\
\hline Frontal lobe (medial) & Callasomarginal artery & & \\
\hline $\begin{array}{l}\text { Brainstem auditory evoked poten- } \\
\text { tials, waves } 4 \& 5\end{array}$ & Peduncular posterior cerebral artery & & \\
\hline Anterior nucleus (thalamus) & Internal cerebral vein & \multirow{5}{*}{$\begin{array}{l}\text { Nowinski et al., 2010; Teplitzky et } \\
\quad \text { al., 2014; computational modeling }\end{array}$} & \multirow[t]{2}{*}{$0.4-1.4 \mathrm{~mm}$} \\
\hline Fornix & Internal cerebral vein & & \\
\hline Nucleus accumbens & $A_{1}$ segment of the anterior cerebral artery & & $2.2-2.6 \mathrm{~mm}$ \\
\hline Subgenual cingulate white matter & $\mathrm{A}_{2}$ segment of the anterior cerebral artery & & $1.9-2.2 \mathrm{~mm}$ \\
\hline Ventral capsule & $\mathrm{A}_{2}$ segment of the anterior cerebral artery & & \\
\hline
\end{tabular}

$M R V=M R$ venography .

*Andrews BT, Dujovny M, Mirchandani HG, Ausman Jl: Microsurgical anatomy of the venous drainage into the superior sagittal sinus. Neurosurgery 24:514-520, 1989.

for endovascular DBS stimulation based on neurovascular modeling. They identified 5 targets near a vessel with an intraluminal diameter $>1 \mathrm{~mm}$, suggesting a viable application of endovascular DBS. They also tested the subgenual cingulate white matter and fornix in a computational model of axons to predict the response to endovascular stimulation. Interestingly they concluded that a ring electrode model (stent-like) was more efficient at neural activation than guidewire designed with the length of the ring electrode not correlating to increased efficacy. The study found that endovascular activation thresholds were comparable to DBS lead activation and that contralateral activation was better via a unilateral endovascular approach compared to DBS lead unilateral modeling. Suboptimal placement did, however, affect activation on the endovascular modeling. ${ }^{49}$

\section{Stentrodes and Possible Responsive Neural Monitoring}

Responsive pacing began with cardiac pacers that use a battery generator to supply leads in the vascular space, responsible for controlling and responding to myocytes and bundle projections. Rates of transvenous lead-induced thrombosis were reported between $0.6 \%$ and $3.5 \% .^{1}$ Intravenous vagal nerve stimulators for cardiac arrhythmias have also been trialed. ${ }^{50}$ Electroencephalographic (EEG) recordings from intravascularly placed electrodes date back to 1973 , described in baboons by Penn et al. ${ }^{43}$ Since that time, endovascular electrode technology has undergone many changes from macro leads, to micro leads on a wire or catheter, and now to nanowire array leads and stentrodes. ${ }^{45}$ Responsive technology was only recently applied to the brain with the advent of NeuroPace's RNS System, a responsive neural pacing system that consists of depth leads or cortical grids surgically implanted via craniotomy that are capable of relaying cortical activity and responding appropriately when epileptic activity is detected via tissue stimulation. The device was shown to decrease partial-onset intractable seizures by $44 \%$ at 1 year and by $54 \%$ at 2 years, in patients in a double-blinded controlled trial. ${ }^{21}$ The system does rely on open craniotomy for implantation, but the generator is recessed in the calvaria, thus decreasing the need for lead tunneling.

Currently patents exist for a wireless cardiac pacing system with intravascularly placed leads, ${ }^{8}$ and it is likely only a matter of time before this technology is incorporated into cranial products. Briefly, the described patent utilizes a radiofrequency generator to transcutaneously and wirelessly stimulate a receiver on the stentrode, which is then able to convert the radiofrequency into an electrical pulse. Thus, in theory, different stentrodes can be controlled by different radiofrequencies. Integral to this minimally invasive approach would be a stentrode and a transcutaneous/transcalvarial wireless method of exciting such a device. Similar studies utilizing an ultrasound generator and transvenously placed receiver electrode (on a catheter) capable of converting ultrasound energy to electrical energy was demonstrated, in 24 people, to be a safe and feasible way of "wireless" cardiac pacing. ${ }^{29}$ Thus, one can explore the concept of placing an electrode on a stent rather than a catheter. The wireless nature of the electrode allows for less thrombogenicity, given the smaller surface area of a foreign body in vasculature and easier, less disturbing placement.

Minimally invasive implantation of cortical recording stents (stentrodes) were reported in 2016 by Oxley et al. ${ }^{42}$ The small stentrode was endovascularly placed in the cortical vein of sheep, with demonstrated recording of cortical activity over 190 days. Venous patency was maintained, and vascular corticography data were comparable to those obtained by epidural arrays. A stentrode 
consisted of platinum discs mounted $2.5 \mathrm{~mm}$ apart on a stent-like device, delivered through a 4-Fr catheter via the jugular vein, with the leads anchored at the jugular access site. A cortical vein near the motor area was used, given its relatively constant colocalization with the motor area. Somatosensory evoked potentials were detectable in $98 \%$ of channels. ${ }^{55}$ Micro-CT and histological evaluation have shown vessel patency and stent strut incorporation into the vessel wall with neointima coverage of $78 \%$ at $2-4$ weeks..$^{40}$ Intravascular electrode recording has also been verified in a swine model to record high-bandwidth, highfidelity electrical correlates of physiological activity. ${ }^{5}$

Thus, two types of stentrodes are reported. One utilizes connecting wires, which must be anchored and tunneled as in standard DBS leads. This type of stentrode is more easily seen as working via a jugular vein puncture, as described by Oxley et al., ${ }^{42}$ which can decrease the amount of intravascular lead necessary and likely will not be suitable for arterial use. The second wireless stentrode would require microchip circuitry with a transmitting and receiving antenna, similar to that as described by Howard.22 Similar devices have been described and implanted in cardiac swine models. One such device is a battery-less, wireless, $4 \times 1-\mathrm{mm}$ device with a microchip and radiofrequency "on chip" antenna capable of harvesting an external radiofrequency signal at $2-\mathrm{cm}$ tissue thickness. Furthermore, it generates a stimulation pulse signal of 1.3 V. ${ }^{47}$ Implantable microchip technology has been trialed in humans previously in the form of a parathyroid drug dispenser that responded wirelessly over medical device frequency. ${ }^{14}$ An external generator or receiver would be necessary. In either case, recording and stimulating capabilities would be present. Such adaptive stimulation has been performed with current DBS leads and braincomputer interfacing, leading to a $56 \%$ reduction in DBS stimulation time in Parkinson's disease patients. ${ }^{31}$

\section{Future Applications of Vascular Stent-Based Technologies}

Brancato et al. have described an intravascular pressure-sensing device utilizing piezoelectric technology. They tested the device in carotid arteries of sheep successfully. ${ }^{6}$ Thus, the ability to develop multimodality stentrodes is feasible, with devices able to sample electrographic, hematological, and pneumatic stresses. One can imagine the application of a pressure-sensing device implanted into the middle meningeal artery as a viable alternative to intracranial pressure monitors, with little risk of symptomatic morbidity if the artery were occluded. Such a device would aid in trauma patients and shunt-treated patients with the possibility of responsive shunting via programmable valve communication.

Similarly, stents can be made to transmit other signals of physiological relevance. RFID STENTag is a stentrode of sorts described by Occhiuzzi et al. as a radiofrequencytagged stent that can monitor the in-stent stenosis of the carotid artery in correlation with the signal strength emitted..$^{38}$

Yanagisawa et al. ${ }^{57}$ utilized surgically implanted subdural electrodes in a poststroke patient and were able to demonstrate real-time control of a prosthetic hand. The authors reported that the onset of the hand movement was detected within 0.37 seconds of the actual movement. After electrocorticography decoding, the brain-machine interface used power modulations of the patient's electrocorticography during hand movement. In a free-run period, the patient's hand movements were faithfully mimicked by the prosthesis. ${ }^{57}$ Stentrodes would allow a craniotomyfree placement of the leads, and one could conceptualize a type of device in which the patient's own arm could be "innervated" with electrodes, allowing for brain-machinebody interfacing. ${ }^{33}$ This type of limb reinnervation could one day help patients with stroke, demyelinating conditions, and spinal cord injury.

Alternating electrical field treatment of tumors, most notably Optune (formerly known as NovoTTF-100A, Novocure Ltd.), has been shown to be efficacious in the treatment of glioblastoma. ${ }^{36}$ The alternating fields are antimitotic. The device is large and cumbersome, and it requires the head to be shaved and numerous electrodes to be connected to the head. This may be yet another field where stentrode-like devices may aid current technology.

Stentrodes have briefly been discussed as above for epilepsy treatment, but there may also one day be a better way of long-term recording allowing the patient to be free of wires, glue, and so on.

The use of stentrodes may also have possible applications in the realm of neurocognitive disorders. Previous studies have explored the use of DBS in hippocampal and medial-temporal regions using open-loop stimulation with stimulation leading to improvements in memory. ${ }^{15,35,48,51}$ Recently, researchers reported the use of closed-loop stimulation of lateral temporal cortex for sensing and stimulating the brain during a memory task in 25 neurosurgical patients undergoing intracranial EEG monitoring for refractory epilepsy, demonstrating improved recall..$^{12}$ The lateral temporal cortex would be available to stentrode placement via the middle meningeal artery, as shown in Table 1.

\section{Conclusions}

Endovascular delivery of neuromodulating devices appears to be a feasible, minimally invasive technique with therapeutic applications in many fields. The catheter-based techniques utilized today are able to reach most desired locations for therapeutic action. Limitations at this time lie in the development of durable and safe endovascular leads and stentrodes with the capability of wireless transmission.

\section{References}

1. Barakat K, Robinson NM, Spurrell RA: Transvenous pacing lead-induced thrombosis: a series of cases with a review of the literature. Cardiology 93:142-148, 2000

2. Binder DK, Rau GM, Starr PA: Risk factors for hemorrhage during microelectrode-guided deep brain stimulator implantation for movement disorders. Neurosurgery 56:722-732, 2005

3. Biondi A, Janardhan V, Katz JM, Salvaggio K, Riina HA, Gobin YP: Neuroform stent-assisted coil embolization of wide-neck intracranial aneurysms: strategies in stent deployment and midterm follow-up. Neurosurgery 61:460-469, 2007 
4. Bly MJ, inventor; Cardiac Pacemakers, Inc., assignee: Neurostimulating lead having a stent-like anchor. US patent 8,412,350B2. April 2, 2013

5. Bower MR, Stead M, Van Gompel JJ, Bower RS, Sulc V, Asirvatham SJ, et al: Intravenous recording of intracranial, broadband EEG. J Neurosci Methods 214:21-26, 2013

6. Brancato L, Keulemans G, Verbelen T, Meyns B, Puers R: An implantable intravascular pressure sensor for a ventricular assist device. Micromachines 7:135, 2016

7. Brinjikji W, Murad MH, Lanzino G, Cloft HJ, Kallmes DF: Endovascular treatment of intracranial aneurysms with flow diverters: a meta-analysis. Stroke 44:442-447, 2013

8. Bulkes C, Beutler AJ, Denker S, inventors; Kenergy, Inc., assignee: Wireless cardiac pacing system with vascular electrode-stents. US patent 6,445,953B1. September 3, 2002

9. Chalouhi N, Chitale R, Starke RM, Jabbour P, Tjoumakaris S, Dumont AS, et al: Treatment of recurrent intracranial aneurysms with the Pipeline Embolization Device. J Neurointerv Surg 6:19-23, 2014

10. Cogan SF, Troyk PR, Ehrlich J, Plante TD, Detlefsen DE: Potential-biased, asymmetric waveforms for charge-injection with activated iridium oxide (AIROF) neural stimulation electrodes. IEEE Trans Biomed Eng 53:327-332, 2006

11. Deuschl G, Schade-Brittinger C, Krack P, Volkmann J, Schäfer H, Bötzel K, et al: A randomized trial of deep-brain stimulation for Parkinson's disease. N Engl J Med 355:896908, 2006

12. Ezzyat Y, Wanda PA, Levy DF, Kadel A, Aka A, Pedisich I, et al: Closed-loop stimulation of temporal cortex rescues functional networks and improves memory. Nat Commun 9:365, 2018

13. Falowski SM, Ooi YC, Bakay RA: Long-term evaluation of changes in operative technique and hardware-related complications with deep brain stimulation. Neuromodulation 18:670-677, 2015

14. Farra R, Sheppard NF, McCabe L, Neer RM, Anderson JM, Santini JT, et al: First-in-human testing of a wirelessly controlled drug delivery microchip. Sci Transl Med 4:122ra121, 2012

15. Fell J, Staresina BP, Do Lam AT, Widman G, Helmstaedter $C$, Elger CE, et al: Memory modulation by weak synchronous deep brain stimulation: a pilot study. Brain Stimul 6:270-273, 2013

16. Feres F, Costa JR Jr, Abizaid A: Very late thrombosis after drug-eluting stents. Catheter Cardiovasc Interv 68:83-88, 2006

17. Fiorella D, Levy EI, Turk AS, Albuquerque FC, Niemann DB, Aagaard-Kienitz B, et al: US multicenter experience with the wingspan stent system for the treatment of intracranial atheromatous disease: periprocedural results. Stroke 38:881-887, 2007

18. Gao X, Liang G, Li Z, Wei X, Hong Q: Complications and adverse events associated with Neuroform stent-assisted coiling of wide-neck intracranial aneurysms. Neurol Res 33:841-852, 2011

19. Greenberg BD, Malone DA, Friehs GM, Rezai AR, Kubu CS, Malloy PF, et al: Three-year outcomes in deep brain stimulation for highly resistant obsessive-compulsive disorder. Neuropsychopharmacology 31:2384-2393, 2006

20. Hariz M: Deep brain stimulation: new techniques. Parkinsonism Relat Disord 20 (Suppl 1):S192-S196, 2014

21. Heck CN, King-Stephens D, Massey AD, Nair DR, Jobst BC, Barkley GL, et al: Two-year seizure reduction in adults with medically intractable partial onset epilepsy treated with responsive neurostimulation: final results of the RNS System Pivotal trial. Epilepsia 55:432-441, 2014

22. Howard MA III, inventor; The University of Iowa Research Foundation, assignee: Wireless prosthetic electrode for the brain. US patent 5,800,535. September 1, 1998
23. Hubble JP, Busenbark KL, Wilkinson S, Penn RD, Lyons K, Koller WC: Deep brain stimulation for essential tremor. Neurology 46:1150-1153, 1996

24. Iosif C, Piotin M, Saleme S, Barreau X, Sedat J, Chau Y, et al: Safety and effectiveness of the Low Profile Visualized Intraluminal Support (LVIS and LVIS Jr) devices in the endovascular treatment of intracranial aneurysms: results of the TRAIL multicenter observational study. J Neurointerv Surg 10:675-681, 2018

25. Ishida S, Shimamoto H, Abe T, Motooka H, Sakurai S, Takayama K, et al: Intracranial EEG recording from intravascular electrodes in patients with temporal lobe epilepsy. Epilepsia 39 (Suppl 5):77, 1998 (Abstract)

26. Kondziolka D, Ong JG, Lee JY, Moore RY, Flickinger JC, Lunsford LD: Gamma Knife thalamotomy for essential tremor. J Neurosurg 108:111-117, 2008 (Erratum in J Neurosurg 108: 635, 2008)

27. Kunieda T, Ikeda A, Mikuni N, Ohara S, Sadato A, Taki W, et al: Use of cavernous sinus EEG in the detection of seizure onset and spread in mesial temporal lobe epilepsy. Epilepsia 41:1411-1419, 2000

28. Lee HM, Park H, Ghovanloo M: A power-efficient wireless system with adaptive supply control for deep brain stimulation. IEEE J Solid State Circuits 48:2203-2216, 2013

29. Lee KL, Lau CP, Tse HF, Echt DS, Heaven D, Smith W, et al: First human demonstration of cardiac stimulation with transcutaneous ultrasound energy delivery: implications for wireless pacing with implantable devices. J Am Coll Cardiol 50:877-883, 2007

30. Lipsman N, Schwartz ML, Huang Y, Lee L, Sankar T, Chapman M, et al: MR-guided focused ultrasound thalamotomy for essential tremor: a proof-of-concept study. Lancet Neurol 12:462-468, 2013

31. Little S, Pogosyan A, Neal S, Zavala B, Zrinzo L, Hariz M, et al: Adaptive deep brain stimulation in advanced Parkinson disease. Ann Neurol 74:449-457, 2013

32. Llinás RR, Walton KD, Nakao M, Hunter I, Anquetil PA: Neuro-vascular central nervous recording/stimulating system: using nanotechnology probes. J Nanopart Res 7:111127,2005

33. Lobel DA, Lee KH: Brain machine interface and limb reanimation technologies: restoring function after spinal cord injury through development of a bypass system. Mayo Clin Proc 89:708-714, 2014

34. Mayberg HS, Lozano AM, Voon V, McNeely HE, Seminowicz D, Hamani C, et al: Deep brain stimulation for treatmentresistant depression. Neuron 45:651-660, 2005

35. Miller JP, Sweet JA, Bailey CM, Munyon CN, Luders HO, Fastenau PS: Visual-spatial memory may be enhanced with theta burst deep brain stimulation of the fornix: a preliminary investigation with four cases. Brain 138:1833-1842, 2015

36. Mittal S, Klinger NV, Michelhaugh SK, Barger GR, Pannullo SC, Juhász C: Alternating electric tumor treating fields for treatment of glioblastoma: rationale, preclinical, and clinical studies. J Neurosurg 128:414-421, 2018

37. Nowinski WL, Chua BC, Volkau I, Puspitasari F, Marchenko Y, Runge VM, et al: Simulation and assessment of cerebrovascular damage in deep brain stimulation using a stereotactic atlas of vasculature and structure derived from multiple 3- and 7-tesla scans. J Neurosurg 113:1234-1241, 2010

38. Occhiuzzi C, Contri G, Marrocco G: RFID STENTag for passive vascular monitoring, in Proceedings of the 5th European Conference on Antennas and Propagation (EUCAP). Piscataway, NJ: IEEE, 2011, pp 3476-3478 (https://ieeexplore.ieee.org/document/5782311/) [Accessed June 14, 2018]

39. Oh MY, Abosch A, Kim SH, Lang AE, Lozano AM: Longterm hardware-related complications of deep brain stimulation. Neurosurgery 50:1268-1276, 2002 
40. Opie NL, van der Nagel NR, John SE, Vessey K, Rind GS, Ronayne SM, et al: Micro-CT and histological evaluation of an neural interface implanted within a blood vessel. IEEE Trans Biomed Eng 64:928-934, 2017

41. Orlov K, Kislitsin D, Strelnikov N, Berestov V, Gorbatykh A, Shayakhmetov T, et al: Experience using pipeline embolization device with Shield Technology in a patient lacking a full postoperative dual antiplatelet therapy regimen. Interv Neuroradiol 24:270-273, 2018

42. Oxley TJ, Opie NL, John SE, Rind GS, Ronayne SM, Wheeler TL, et al: Minimally invasive endovascular stent-electrode array for high-fidelity, chronic recordings of cortical neural activity. Nat Biotechnol 34:320-327, 2016

43. Penn RD, Hilal SK, Michelsen WJ, Goldensohn ES, Driller J: Intravascular intracranial EEG recording. Technical note. J Neurosurg 38:239-243, 1973

44. Rangel-Castilla L, Cress MC, Munich SA, Sonig A, Krishna $\mathrm{C}, \mathrm{Gu}$ EY, et al: Feasibility, safety, and periprocedural complications of pipeline embolization for intracranial aneurysm treatment under conscious sedation: University at Buffalo Neurosurgery Experience. Neurosurgery 11 (Suppl 3):426430,2015

45. Sefcik RK, Opie NL, John SE, Kellner CP, Mocco J, Oxley TJ: The evolution of endovascular electroencephalography: historical perspective and future applications. Neurosurg Focus 40(5):E7, 2016

46. Stoeter P, Dieterle L, Meyer A, Prey N: Intracranial electroencephalographic and evoked-potential recording from intravascular guide wires. AJNR Am J Neuroradiol 16:12141217, 1995

47. Sun Y, Greet B, Burkland D, John M, Razavi M, Babakhani A: Wirelessly powered implantable pacemaker with on-chip antenna, in 2017 IEEE MTT-S International Microwave Symposium (IMS). Piscataway NJ: IEEE, 2017, pp 12421244 (https://ieeexplore.ieee.org/document/8058831/) [Accessed June 14, 2018]

48. Suthana N, Haneef Z, Stern J, Mukamel R, Behnke E, Knowlton B, et al: Memory enhancement and deep-brain stimulation of the entorhinal area. N Engl J Med 366:502510,2012

49. Teplitzky BA, Connolly AT, Bajwa JA, Johnson MD: Computational modeling of an endovascular approach to deep brain stimulation. J Neural Eng 11:026011, 2014

50. Thompson GW, Levett JM, Miller SM, Hill MR, Meffert WG, Kolata RJ, et al: Bradycardia induced by intravascular versus direct stimulation of the vagus nerve. Ann Thorac Surg 65:637-642, 1998

51. Titiz AS, Hill MRH, Mankin EA, M Aghajan Z, Eliashiv D,
Tchemodanov N, et al: Theta-burst microstimulation in the human entorhinal area improves memory specificity. eLife 6:6, 2017

52. Wallace MP, Garabedian RJ, Abrams RM, inventors; Scimed Life Systems, Inc., assignee: Method of intravascularly delivering stimulation leads into brain. US patent application 2005/0137646A1. June 23, 2005

53. Wallace MP, Garabedian RJ, Abrams RM, inventors; Boston Scientific Scimed, Inc., assignee: Method of intravascularly delivering stimulation leads into brain to stimulate the SPG. US patent 7,818,063B2. October 19, 2010

54. Watanabe H, Takahashi H, Nakao M, Walton K, Llinás RR: Intravascular neural interface with nanowire electrode. Electron Commun Jpn 92:29-37, 2009

55. Weiner GM, Ozpinar A, Ducruet A: Endovascular access for cortical mapping. Neurosurgery 79:N22-N23, 2016

56. Wong YT, Opie NL, John SE, Gerboni G, Rind GS, Ronayne SM, et al: Suitability of nitinol electrodes in neural prostheses such as endovascular neural interfaces. Conf Proc IEEE Eng Med Biol Soc 2016:4463-4466, 2016

57. Yanagisawa T, Hirata M, Saitoh Y, Goto T, Kishima H, Fukuma R, et al: Real-time control of a prosthetic hand using human electrocorticography signals. J Neurosurg 114:17151722,2011

58. Young RF, Li F, Vermeulen S, Meier R: Gamma Knife thalamotomy for treatment of essential tremor: long-term results. J Neurosurg 112:1311-1317, 2010

\section{Disclosures}

The authors report no conflict of interest concerning the materials or methods used in this study or the findings specified in this paper.

\section{Author Contributions}

Conception and design: Rajah, Saber. Acquisition of data: Rajah, Saber, Singh. Analysis and interpretation of data: Rajah, Saber, Singh. Drafting the article: Rajah. Critically revising the article: Rajah, Saber, Singh. Reviewed submitted version of manuscript: Rajah, Saber, Singh. Approved the final version of the manuscript on behalf of all authors: Rangel-Castilla. Administrative/techni$\mathrm{cal} /$ material support: Rangel-Castilla. Study supervision: RangelCastilla.

\section{Correspondence}

Leonardo Rangel-Castilla: Mayo Clinic, Rochester, MN. rangelcastilla.leonardo@mayo.edu. 